\title{
Clinical Characteristics of Pandemic Influenza A (H1N1) 2009 Pediatric Infection in Busan and Gyeongsangnam-do: One Institution
}

\author{
Myung Chul Lee, M.D. ${ }^{1}$, Hye Young Kim, M.D. ${ }^{2}$, Seom Gim Kong, M.D. ${ }^{2}$, Young Mi Kim, M.D. ${ }^{2}$, Su Eun Park, \\ M.D. ${ }^{2}$, Young Tak Im, M.D. ${ }^{2}$, Hee Ju Park, M.D. ${ }^{2}$ \\ ${ }^{1}$ Department of Pediatrics, Seoul National University College of Medicine, Seoul, ${ }^{2}$ Department of Pediatrics, Pusan National \\ University College of Medicine, Busan, Korea
}

Background: This study investigated the clinical characteristics and risk factors of the severity of pandemic influenza A (H1N1) 2009 infection in pediatric patients in Busan and Gyeongsangnam-do.

Methods: Cases of influenza A (H1N1) 2009 in patients under the age of 18 years, confirmed by reverse transcription polymerase chain reaction, at Pusan National University Hospital and Pusan National University Yangsan Hospital from the last week of August 2009 through the last week of February 2010 were retrospectively analyzed.

Results: Of the 3,777 confirmed cases of influenza A (H1N1) 2009, 2,200 (58.2\%) were male and 1,577 (41.8\%) were female. The average age of the patients was $8.4 \pm 4.8$ years. The total cases peaked during 44th to 46th week. Most of the patients were in the 5- to 9-year-old age group. Oseltamivir was administered to 2,959 (78.3\%) of the patients. 221 patients (5.9\%) were hospitalized, age an average of $6.7 \pm 4.5$ years. The average duration of hospitalization was $7.4 \pm 5.6$ days. One hundred cases $(45.2 \%)$ had pneumonia. Risk factors for hospitalization included male gender, $<2$ years of age, and underlying disease. Children with asthma were at very high risk of hospitalization, over 20 times the non-asthmatic children (odds ratio [OR], 21.684; confidence interval [CI], 13.295 39.791). Likewise the children with neurologic deficits faced a 16 times higher risk (OR, 15.738; CI, 7.961 31.111). Ten of the patients (4.5\%) were admitted to the intensive care unit, and eight $(3.6 \%)$ required mechanical ventilation.

Conclusion: Of the pediatric patients with pandemic influenza A (H1N1) 2009, most of the patients were in the 5- to 9-year-old age group. Risk factors for hospitalization included male gender, $<2$ years of age, and underlying disease. The most common complication was pneumonia. The very high risk of severe morbidity in children with asthma or neurologic disease shows the critical importance of targeted vaccine coverage, special awareness and swift care by both guardians and primary care providers.

Key Words: Influenza A Virus, H1N1 Subtype; Epidemiologic Study Characteristics as Topic; Pediatrics

\section{Introduction}

With the outbreak of the influenza $\mathrm{A}(\mathrm{H} 1 \mathrm{~N} 1)$ in the US and Mexico in March 2009, World Health Organization (WHO) declared it as pandemic influenza in June 2009 for the first time in 41 years $^{1}$. A total of 18,449

Address for correspondence: Young Tak Im, M.D. Department of Pediatrics, Pusan National University College of Medicine, 179, Gudeok-ro, Seo-gu, Busan 602-739, Korea Phone: 82-51-240-7298, Fax: 82-51-248-6205

E-mail: limyt@pusan.ac.kr

Received: Feb. 20, 2012

Revised: Mar. 29, 2012

Accepted: May 1, 2012 patients died of pandemic influenza A (H1N1) 2009 in 214 countries $^{2}$. In Korea, a total of 270 patients died of the influenza between the period of April 2009 and August 2010 after the first patient was confirmed in May $2009^{3}$.

Characteristics of pandemic influenza A (H1N1) 2009 include high incidence in infants and young patients, and high rate of admission to hospital compared with those of seasonal influenza ${ }^{4}$. In the present study, epidemiological and clinical characteristics of pandemic influenza A (H1N1) 2009 pediatric patients in Busan and Gyeongsangnam-do area were analyzed, and risk factors of the pediatric patients in intensive care unit (ICU) 
were investigated.

\section{Materials and Methods}

\section{Subjects}

Patient records of 3,777 patients who were in the age of 18 or younger, and definitely diagnosed with pandemic influenza A (H1N1) 2009 in Pusan National University Hospital and Pusan National University Yangsan Hospital over the period of 27 weeks between the last week of August 2009 and the last week of February 2010 were retrospectively analyzed. Throat swabs of the patients who visited the hospitals with complaints of similar symptoms to influenza were collected from oral pharynx or nasopharynx and then, real-time reverse transcription polymerase chain reaction (RT-PCR) was tested before making definite diagnoses.

\section{Methods}

1) Real-time RT-PCR assay: Using MagNa Pure LC 2.0 (Roche Diagnostics Ltd., Basel, Switzerland), RNA was separated. Complementary DNA (cDNA) was synthesized using Revertaid First Strand cDNA Synthesis Kit (Fermentas UAB, Vilnius, Lithuania), and was amplified through ABI 7500 (Applied Biosystems, Foster City, CA, USA) to investigate subtypes of influenza.

RNA-separated samples were tested using primer/ probe sets of universal influenza type A (InfA), swine influenza A (swInfA), swine H1 influenza (swH1), and RNaseP. When both RNaseP and InfA probe were positive, and the positive result of either swInfA or swH1 probe was available within 40 cycles, pandemic influenza A (H1N1) 2009 was confirmed positive.

2) Data collection: Through patient records, information such as gender, age, date of making a definite diagnosis, symptoms, complete blood count, general chemistry, chest X-ray, comorbid diseases, underlying diseases and treatments was collected, and risk factors of inpatients and patients in ICU were investigated.

Fever was defined as $37.8^{\circ} \mathrm{C}$ or higher of tympanic temperature. In complete blood counts, leukopenia was defined as less than $5,000 / \mu \mathrm{L}$, lymphopenia as the ab- solute lymphocyte count at less than $800 / \mu \mathrm{L}$, or the lymphocyte ratio at less than $15 \%$, and thrombocytopenia as less than $150,000 / \mu \mathrm{L}$. In general chemistry, cases of C-reactive protein (CRP) exceeding $0.5 \mathrm{mg} / \mathrm{dL}$, aspartate aminotransferase (AST) exceeding $100 \mathrm{IU} / \mathrm{L}$, and alanine aminotransferase (ALT) exceeding 100 IU/L were defined as "increase."

Chest X-ray included simple chest X-ray and computed tomography, and findings of diagnostic images were reviewed by radiologists of the hospital.

3) Statistics: SPSS version 18.0 (SPSS Inc., Chicago, IL, USA) was used for statistical processing. T-test and Mann- Whitney test were used for mean value comparison. In order to find out relationship between single independent variable and hospital admission, chi-square test was used. In order to find out relationship between multiple independent variables and hospital admission, multivariate binominal logistic regression analysis was used to obtain odds ratios (OR) and 95\% confidence interval (CI). When OR was larger than 1.0, probability of hospital admission was considered higher than reference group. When p-value was less than 0.05 , statistical significance was recognized.

\section{Results}

\section{Characteristics of patients and incidence}

Number of patients who were 18 years of age or younger, and diagnosed with pandemic influenza A (H1N1) 2009 was 3,777 including 1,053 in Pusan National University Hospital, and 2,724 in Pusan National University Yangsan Hospital. Number of male patients was 2,200 (58.2\%), and of female patients was

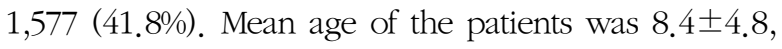
and the male mean age of $8.5 \pm 5.0$ was significantly higher than the female mean age of $8.2 \pm 4.6(\mathrm{p}<0.05)$.

In terms of incidence, less than 100 cases per week were reported between the 36th week and the 42nd week of 2009, but the rate abruptly increased from the $43 \mathrm{rd}$ week reaching a peak in the 44 th $\sim 46$ th week. From the 47th week, the incidence decreased, and the incidence was less than 10 per week since the 2nd 
week of 2010 (Figure 1). The group of 15 18 of age showed an abrupt increase in incidence in August and September 2009, and a peak in October 2009, while all other groups of less than 14 years of age showed an abrupt increase in incidence in October 2009, and a peak in November 2009 meaning one month delay in the incidence pattern. Particularly, the $5 \sim 9$ years of age group reported more than 2 times of incidence rate than other groups in November 2009. In and after December 2009, number of patients abruptly decreased in all age groups, but the 4 years and younger age group reported the least decrease rate among other age groups and ac-

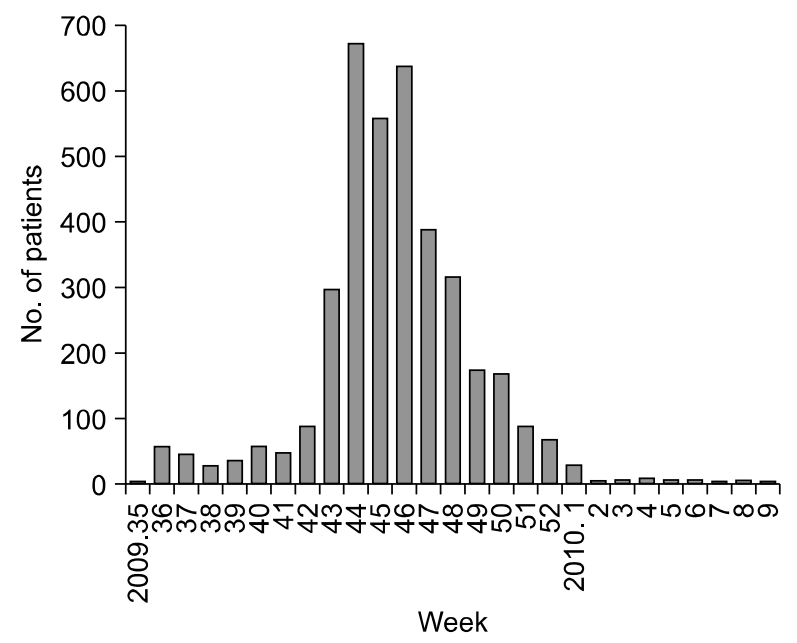

Figure 1. Distribution of patients with pandemic influenza A (H1N1) 2009 by weeks.

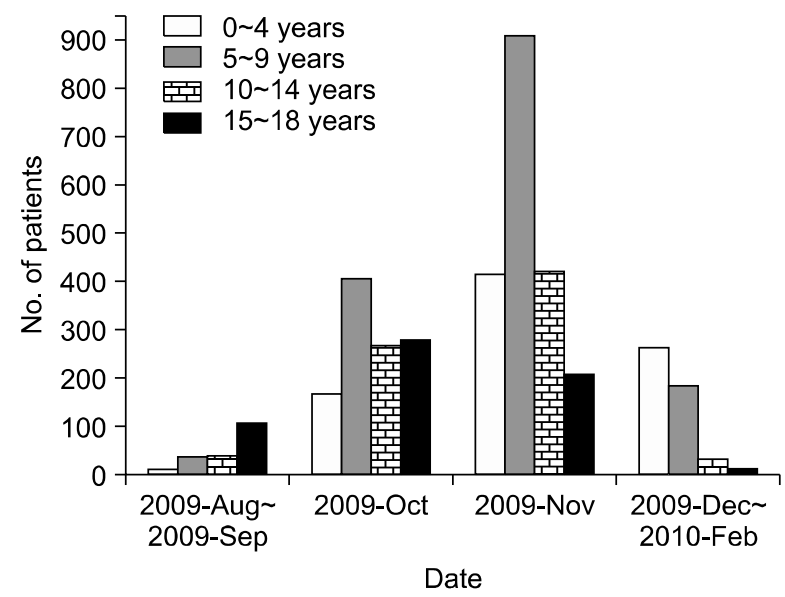

Figure 2. Distribution of patients with pandemic influenza A (H1N1) 2009 by months and age groups. cordingly, the group showed the largest number of patients during the period (Figure 2).

A total of 219 patients (5.8\%) were confirmed to have underlying diseases. Asthma formed the largest share with 62 patients (28.3\%) followed by neurologic diseases in 39 patients $(17.8 \%)$, chronic heart diseases in 23 patients (10.5\%), neoplastic diseases in 15 patients (6.8\%), and kidney diseases in 14 patients (6.4\%).

\section{Symptoms and signs}

In terms of respiratory symptoms, fever was found in the most 3,218 patients (85.2\%) followed by coughing in 2,828 patients $(74.9 \%)$, rhinorrhea in 1,438 patients

Table 1. Clinical manifestations of patients with pandemic influenza A (H1N1) 2009

\begin{tabular}{|c|c|}
\hline Symptoms and signs & No. (\%) $(n=3,777)$ \\
\hline \multicolumn{2}{|l|}{ Respiratory symptoms and signs } \\
\hline Fever & $3,218(85.2)$ \\
\hline Cough & $2,828(74.9)$ \\
\hline Rhinorrhea & $1,438(38.1)$ \\
\hline Sore throat & $968(25.6)$ \\
\hline Sputum & $563(14.9)$ \\
\hline Dyspnea & $112(3.0)$ \\
\hline Hoarseness & $6(0.2)$ \\
\hline \multicolumn{2}{|c|}{ Gastric and intestinal symptoms and signs } \\
\hline Vomiting & $175(4.6)$ \\
\hline Nausea & $99(2.6)$ \\
\hline Abdominal pain & $99(2.6)$ \\
\hline Anorexia & 87 (2.3) \\
\hline Diarrhea & $63(1.7)$ \\
\hline \multicolumn{2}{|l|}{ Neurologic symptoms and signs } \\
\hline Headache & $576(15.3)$ \\
\hline Dizziness & $54(1.4)$ \\
\hline Seizure & $20(0.5)$ \\
\hline Decreased mentality & $19(0.5)$ \\
\hline Delirium & $4(0.1)$ \\
\hline \multicolumn{2}{|l|}{ Systemic symptoms and signs } \\
\hline Myalgia & $195(5.2)$ \\
\hline Rash & $12(0.3)$ \\
\hline Petechiae & $2(0.1)$ \\
\hline \multicolumn{2}{|c|}{ Cardiovascular symptoms and signs } \\
\hline Chest pain & $10(0.3)$ \\
\hline \multicolumn{2}{|c|}{ Head and neck symptoms and signs } \\
\hline Epistaxis & $5(0.1)$ \\
\hline Ophthalmodynia & $4(0.1)$ \\
\hline Conjunctival injection & $2(0.1)$ \\
\hline None & $14(0.4)$ \\
\hline
\end{tabular}


(38.1\%), sore throat in 968 patients $(25.6 \%)$, sputum in 563 patients (14.9\%), dyspnea in 112 patients (3.0\%), and hoarseness in 6 patients $(0.2 \%)$. In terms of digestive symptoms, vomiting was found in 175 patients (4.6\%) followed by nausea in 99 patients $(2.6 \%)$, abdominal pain in 99 patients $(2.6 \%)$, anorexia in $87 \mathrm{pa}-$ tients $(2.3 \%)$, and diarrhea in 63 patients $(1.7 \%)$. In terms of neurologic symptoms, headaches were found in 576 patients $(15.3 \%)$, dizziness in 54 patients $(1.4 \%)$, seizure in 20 patients $(0.5 \%)$, decreased mentality in 19 patients $(0.5 \%)$, and delirium in 4 patients $(0.1 \%)$. Other symptoms included myalgia in 195 patients (5.2\%), rash in 12 patients $(0.3 \%)$, petechiae in 2 patients $(0.1 \%)$, chest pain in 10 patients $(0.3 \%)$, nasal bleeding in 5 patients $(0.1 \%)$, ophthalmodynia in 4 patients $(0.1 \%)$, and conjunctival injection in 2 patients $(0.1 \%)$. Fourteen patients $(0.4 \%)$ reported no symptoms (Table 1$)$.

\section{Blood and radiologic tests}

Out of the total 3,777 patients, blood tests were conducted in 535 patients (14.2\%). Two patients had general chemistry while the rest of 533 patients had both complete blood count and general chemistry. In complete blood count, leukopenia was found in 78 patients (14.6\%), lymphopenia in 263 patients (49.3\%), and thrombocytopenia in 40 patients $(7.5 \%)$. In general chemistry, CRP increased in 377 patients (70.5\%), AST in 16 patients (3.0\%), and ALT in 7 patients (1.3\%).

Simple chest X-ray was conducted in 591 patients (15.6\%). Normal findings were observed in 442 patients (74.8\%), pneumonia in 119 patients $(20.1 \%)$, pleural effusion in 12 patients $(2.0 \%)$, pneumomediastinum in 3 patients $(0.5 \%)$, and respiratory distress syndrome in 2 patients $(0.3 \%)$. Increased perihilar infiltration was observed in 27 patients (4.6\%), overexpansion in 2 patients $(0.3 \%)$, and pulmonary edema in 2 patients $(0.3 \%)$. Computed tomography scans were conducted in 30 patients $(0.8 \%)$ and as a result, pneumonia was observed in 28 patients $(93.3 \%)$, pleural effusion in 7 patients $(23.3 \%)$, pneumomediastinum in 3 patients $(10.0 \%)$, and respiratory distress syndrome in 1 patient $(3.3 \%)$.

\section{Treatments}

Number of patients who received antiviral agents or antibiotics treatments was 2,996 (79.3\%). Among them, 2,789 patients (93.1\%) were administered with antiviral agents, 37 patients (1.2\%) with antibiotics only, and 170 patients $(5.7 \%)$ with both antiviral agents and antibiotics. The antiviral agent administered to the patients was oseltamivir (Tamiflu ${ }^{\circledR}$, Roche, Welwyn Garden City, UK). Antibiotics were administered to 282 patients, and the most frequently used antibiotics was aminopenicillin antibiotics (amoxicillin, ampicillin) which was administered to 162 patients $(57.4 \%)$. Penicillinase-resistant penicillin (nafcillin) was administered to 5 patients $(1.8 \%)$, 3rd generation cephalosporin antibiotics (cefotaxime, ceftriaxone) to 48 patients (17.0\%), macrolide antibiotics (clarithromycin, azithromycin) to $55 \mathrm{pa}-$ tients $(19.5 \%)$, vancomycin to 6 patients $(2.1 \%)$, and carbapenem antibiotics (meropenem) to 2 patients $(0.7 \%)$. Fluconazole which is an antifungal agent was used in 1 patient $(0.4 \%)$.

\section{Characteristics of inpatients and risk assessments}

Number of inpatients was 221 consisting 5.9\% of the total patients. Mean hospital stay was $7.4 \pm 5.6$ days. Mean age of the inpatients was 6.7 \pm 4.5 . Number of male patients was $151(68.3 \%)$ with a mean age of

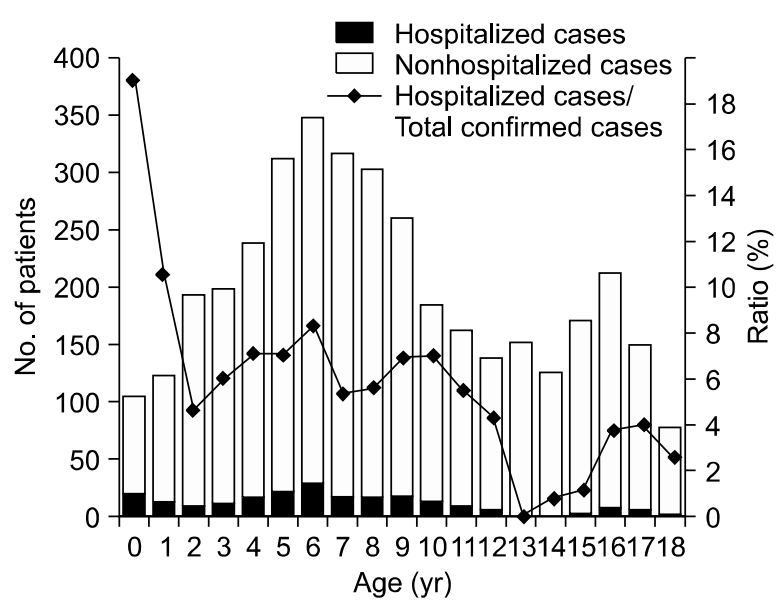

Figure 3. Distribution of patients with pandemic influenza A (H1N1) 2009 by age. 
Table 2. Risks of hospitalization with pandemic influenza A (H1N1) 2009

\begin{tabular}{|c|c|c|c|c|}
\hline & Hospitalized cases $(n=3,777)$ & Nonhospitalized cases $(n=3,777)$ & OR & $95 \% \mathrm{Cl}$ \\
\hline \multicolumn{5}{|l|}{ Sex } \\
\hline Male & 151 & 2,049 & 1.454 & $1.070 \sim 1.975$ \\
\hline Female & 70 & 1,507 & & \\
\hline \multicolumn{5}{|l|}{ Age, yr } \\
\hline$<2$ & 33 & 195 & 3.396 & $2.239 \sim 5.150$ \\
\hline$\geq 2$ & 188 & 3,361 & & \\
\hline \multicolumn{5}{|l|}{ Asthma } \\
\hline Present & 33 & 29 & 21.684 & $13.295 \sim 38.791$ \\
\hline Absent & 188 & 3,527 & & \\
\hline \multicolumn{5}{|c|}{ Neurologic disease } \\
\hline Present & 19 & 20 & 15.738 & $7.961 \sim 31.111$ \\
\hline Absent & 202 & 3,536 & & \\
\hline
\end{tabular}

OR: odds ratio; $\mathrm{Cl}$ : confidence interval.

$6.8 \pm 4.1$, and that of female was 70 patients $(31.7 \%)$ with a mean age of $6.3 \pm 5.2$ showing no statistical difference.

Eight hundred sixty patients (22.8\%) were in the age of 4 or younger, 1,540 patients $(40.8 \%)$ in 5 9, 765 patients $(20.3 \%)$ in $10 \sim 14$, and 612 patients $(16.2 \%)$ in $15 \sim 18$. In terms of inpatients, 71 subjects $(32.1 \%)$ were in the age of 4 or younger, 103 subjects (46.6\%) in 5 9, 29 subjects $(13.1 \%)$ in 10 14, and 18 subjects (8.1\%) in $15 \sim 18$. Patients of younger than 1 year old showed the highest rate of admission to total number of each age group (Figure 3).

Diagnoses at the time of admission included lower respiratory infection in 107 patients (48.4\%), pneumonia in 100 patients (45.2\%), asthma attack in 5 patients (2.3\%), and bronchiolitis in 4 patients (1.8\%). Upper respiratory infection was found in 84 patients (38.0\%), febrile convulsion in 8 patients $(3.6 \%)$, acute gastroenteritis in 4 patients $(1.8 \%)$, acute respiratory distress syndrome in 2 patients $(0.9 \%)$, pneumomediastinum in 2 patients $(0.9 \%)$, hepatitis in 2 patients $(0.9 \%)$, encephalitis in 1 patient $(0.5 \%)$, myelitis 1 patient $(0.5 \%)$, and sepsis in 1 patient $(0.5 \%)$.

Among inpatients, underlying diseases were found in 84 patients (38.0\%) including asthma in 33 patients (14.9\%) followed by neurologic diseases in 19 patients (8.6\%).

In order to investigate risk factors of hospital admis- sion, effects of the 3 independent variable of gender, age and underlying diseases were analyzed. As a result, male (OR, 1.454; 95\% CI, 1.070 1.975), 2 years of age or younger (OR, 3.396; 95\% CI, 2.239 5.150), asthma (OR, 21.684; 95\% CI, 13.295 38.791), and neurologic diseases (OR, 15.738; 95\% CI, 7.961 31.111) were the statistically significant risk factors of hospital admission (Table 2). Factors such as leukopenia, lymphopenia, and increase in CRP did not show significant relations with hospital admission.

Regarding treatments on inpatients, 50 subjects (22.6\%) were administered with antiviral agents only, 27 subjects (12.2\%) with antibiotics only, and 127 subjects (57.5\%) with both antiviral agents and antibiotics. A total of 228 patients were prescribed with antibiotics including aminopenicillin antibiotics with 132 patients (57.9\%), penicillinase-resistant penicillin with 5 patients (2.2\%), cephalosporin antibiotics with 47 patients (20.6\%), macrolide antibiotics with 33 patients (14.5\%), aminoglycoside antibiotics with 2 patients (0.9\%), vancomycin with 6 patients $(2.6 \%)$, and carbapenem antibiotics with 2 patients (0.9\%). Fluconazole which is an antifungal agent was used for 1 patient $(0.4 \%)$.

\section{Characteristics and progress of pediatric patients in ICU}

ICU treatments were performed for 10 patients (8 males and 2 females) or $0.3 \%$ of the total. Mean age 


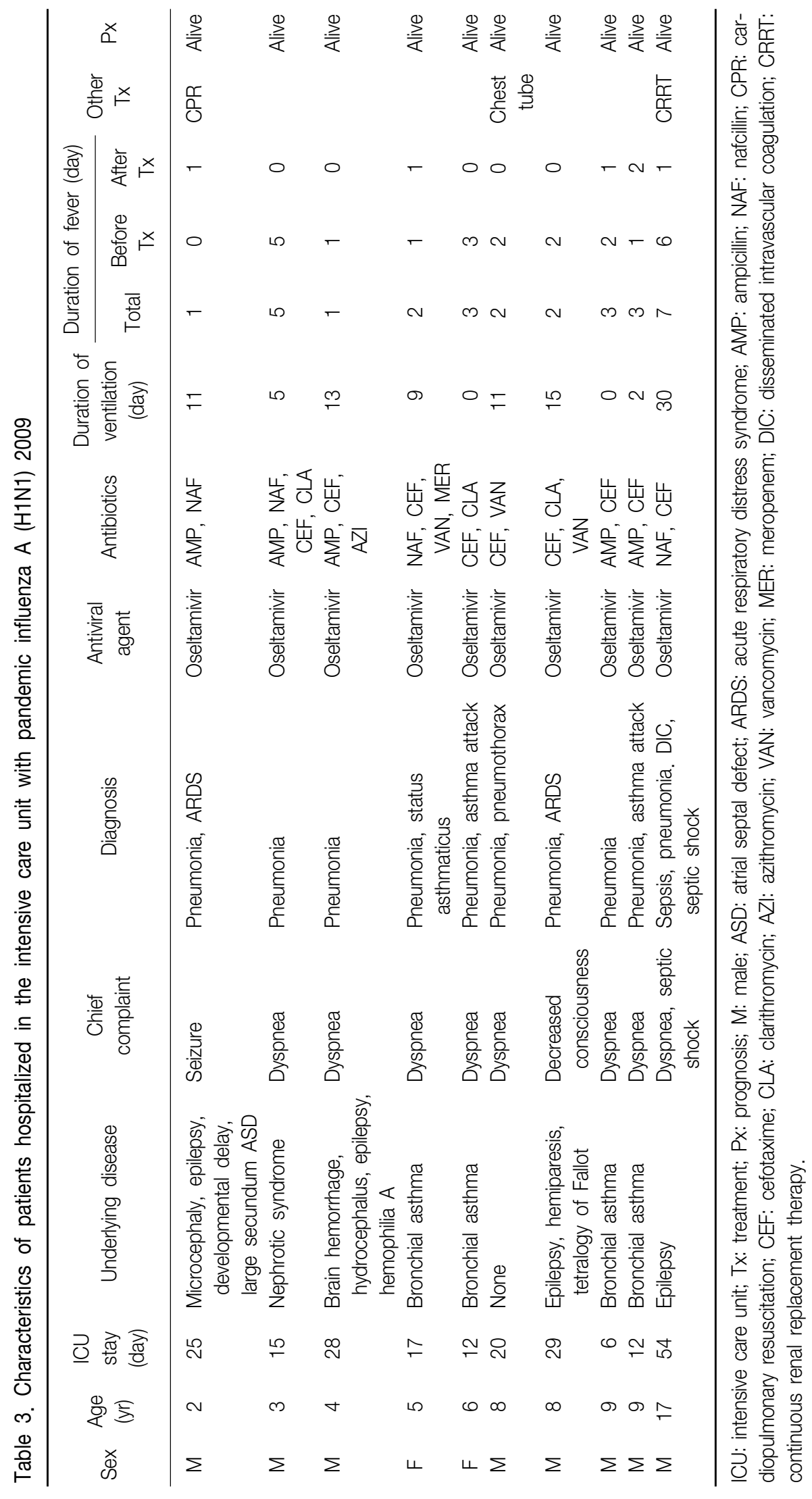


was $7.1 \pm 4.3$ showing no statistical difference from the rest of the patients. Mean days of ICU stay were 21.8 \pm 13.6 . Major symptoms included dyspnea in 8 patients $(80 \%)$, seizure in 1 patient (10\%), and decreased mentality in 1 patient (10\%). Diagnoses were pneumonia in 10 patients (100\%), asthma attack in 3 patients (30\%), acute respiratory distress syndrome in 2 patients (20\%), and sepsis and disseminated intravascular coagulopathy in 1 patient (10\%), respectively. As underlying diseases, asthma was observed in 4 patients (40\%), neurologic diseases (epilepsy, paralysis, hydrocephalus, and developmental disorder) in 4 patients (40\%), and nephrotic syndrome in 1 patient (10\%). One patient (10\%) did not have an underlying disease. In hematologic test, leukopenia was observed in 1 patient (10\%), lymphopenia in 8 patients (80\%), thrombocytopenia in 1 patient (10\%), increase in CRP in 9 patients (90\%), and increase in AST in 1 patient (10\%). No patient reported an increase in ALT. All patients were administered with oseltamivir plus 2 or more of antibiotics. Mechanical ventilators were used for 8 patients (80\%) for a mean of $9.6 \pm 9.0$ days, and 1 patient (10\%) received continuous renal replacement therapy. No patient died after ICU treatments (Table 3).

\section{Discussion}

Despite the present study was conducted by a single-institution, a large number of 3,777 patients who were treated in the representative hospital of Busan and Gyeongsangnam-do area participated in the study, and data representing the area were obtained. Considering the most young adults or pediatric patients of pandemic influenza A (H1N1) 2009, the present study is expected to sufficiently reflect characteristics of pandemic influenza A (H1N1) 2009 infection.

According to the present study, risk factors of hospital admission were male, 2 years of age or younger, and underlying diseases of asthma and neurologic diseases. Ratio of male patients to all patients was $58.2 \%$, and the male ratio of hospital admission was $68.3 \%$ meaning male gender was a statistically significant risk factor of hospital admission. In precedent study on pediatric inpatients, ratio of male was reported at $54 \sim 60 \% \%^{5-8}$ showing a larger share than female. However, these studies neither analyzed the gender difference, nor discussed about the effect of gender to disease severity. Additional studies on gender effects may be necessary in future.

In terms of age, incidence rate was the highest in the 5 9 years of age group. By comparison, rate of admission to total number of each age group was highest in the group of 1 year of age or younger, and 2 years of age or younger was confirmed to be a statistically significant risk factor of hospital admission. According to precedent studies, incidence rate is highest in school age and adolescent patients ${ }^{9}$, and probability of hospital admission is high in infant and preschool age patients corresponding to the results of the present study. According to the statistics of US Centers for Disease Control and Prevention (CDC) regarding an accumulative hospitalization rate in 100,000 patients, $0 \sim 4$ age group showed a $2 \sim 3$ times higher rate of admission than other age groups ${ }^{10}$. Report from New Zealand also shows the highest rate of admission in the group of 5 years of age or younger ${ }^{11}$.

Regarding age distribution associated with death, death rate of infants is lower than the other age groups. According to US CDC, group of 5 18 age showed the lowest death rate followed by $0 \sim 4$ age group ${ }^{10}$. Similar results were reported by Korea $\mathrm{CDC}^{3}$, and no death was reported in the present study. By contrast, elderly patients showed a high death rate. In an American study, $50 \sim 64$ age group showed the highest death rate ${ }^{10}$. Korea CDC reported the highest death rate in the group of 70 years of age or older ${ }^{3}$.

Among underlying diseases, presence of asthma and neurologic diseases increases probability of hospital admission. In the present study, $14.9 \%$ of inpatients had asthma as underlying disease, while $8.6 \%$ had neurologic diseases. These two diseases were confirmed to be statistically significant risk factors of hospital admission. Precedent studies also reported asthma and neurologic diseases as risk factors of hospital admission in pediatric patients ${ }^{5,12-15}$. According to studies, asthma was 
most found in $22 \sim 37 \%$ of inpatients as an underlying disease $^{5,12-15}$. In particular, a Canadian study reported that asthma was more important risk factor of hospital admission compared with the case of seasonal influenza, and even mild asthma was a risk factor ${ }^{5}$. Neurologic diseases was also an underlying disease that $17 \sim 22 \%$ of pediatric inpatients had, ${ }^{5,12}$.

The most common complication of pandemic influenza A (H1N1) 2009 was pneumonia. In the present study, 45.3\% of inpatients experienced pneumonia. According to a Canadian study, $37 \%$ of pediatric inpatients had pneumonia as a comorbid disease ${ }^{15}$. In an American study, pneumonia was accompanied in 55\% of dead patients $^{16}$.

In conclusion, group of 5 9 years of age formed the largest share of patients, and probability of hospital admission was high in case of male, 2 years of age or younger, and presence of asthma and neurologic diseases. Common symptoms included fever, coughing, rhinorrhea, sore throat and headaches. Pneumonia was the most frequent complication. Since the present study was conducted in the area of Busan and Gyeongsangnam-do, large scale multicenter studies will be necessary to understand Korean pandemic influenza A (H1N1) 2009 better.

\section{References}

1. World Health Organization. World now at the start of 2009 influenza pandemic. Geneva: World Health Organization; [cited 2011 Dec 10]. Available from: http://who.int/mediacentre/news/statements/2009/ h1n1_pandemic_phase6_20090611/en/index.html.

2. World Health Organization. Pandemic (H1N1) 2009: update 112. Geneva: World Health Organization; [cited 2009 Dec 10]. Available from: http://www.who.int/ csr/don/2010_08_06/en/.

3. Korea Centers for Disease Control and Prevention. Analysis of reported pandemic influenza (A/H1N1 2009) virus infections in Korea: from April, 2009 through August, 2010. Public Health Wkly Rep 2010; 38:637-42.

4. Kim WJ. Epidemiologic and clinical characteristics of pandemic influenza (1918-2009). Infect Chemother 2009; 41 Suppl 2:5.
5. O'Riordan S, Barton M, Yau Y, Read SE, Allen U, Tran D. Risk factors and outcomes among children admitted to hospital with pandemic H1N1 influenza. CMAJ 2010;182:39-44.

6. Libster R, Bugna J, Coviello S, Hijano DR, Dunaiewsky M, Reynoso N, et al. Pediatric hospitalizations associated with 2009 pandemic influenza A (H1N1) in Argentina. N Engl J Med 2010;362:45-55.

7. Bettinger JA, Sauvé LJ, Scheifele DW, Moore D, Vaudry W, Tran D, et al. Pandemic influenza in Canadian children: a summary of hospitalized pediatric cases. Vaccine 2010;28:3180-4.

8. Da Dalt L, Chillemi C, Cavicchiolo ME, Bressan S, Calistri A, Palù $G$, et al. Pandemic influenza A (H1N1v) infection in pediatric population: a multicenter study in a north-east area of Italy. Ital J Pediatr 2011;37:24.

9. Carcione D, Giele C, Dowse GK, Mak DB, Goggin L, Kwan K, et al. Comparison of pandemic (H1N1) 2009 and seasonal influenza, Western Australia, 2009. Emerg Infect Dis 2010;16:1388-95.

10. Jhung MA, Swerdlow D, Olsen SJ, Jernigan D, Biggerstaff M, Kamimoto L, et al. Epidemiology of 2009 pandemic influenza A (H1N1) in the United States. Clin Infect Dis 2011;52 Suppl 1:S13-26.

11. Bandaranayake D, Jacobs M, Baker M, Hunt D, Wood T, Bissielo A, et al. The second wave of 2009 pandemic influenza $\mathrm{A}(\mathrm{H} 1 \mathrm{~N} 1)$ in New Zealand, JanuaryOctober 2010. Euro Surveill 2011;16. pii:19788.

12. Jain S, Kamimoto L, Bramley AM, Schmitz AM, Benoit SR, Louie J, et al. Hospitalized patients with 2009 H1N1 influenza in the United States, April-June 2009. N Engl J Med 2009;361:1935-44.

13. Bagdure D, Curtis DJ, Dobyns E, Glodé MP, Dominguez SR. Hospitalized children with 2009 pandemic influenza A (H1N1): comparison to seasonal influenza and risk factors for admission to the ICU. PLOS One 2010;5:e15173.

14. Okada T, Morozumi M, Matsubara K, Komiyama O, Ubukata K, Takahashi T, et al. Characteristic findings of pediatric inpatients with pandemic (H1N1) 2009 virus infection among severe and nonsevere illnesses. J Infect Chemother 2011;17:238-45.

15. Launay E, Ovetchkine P, Saint-Jean M, Coïc L, Ducruet $\mathrm{T}$, Charest H, et al. Novel influenza A (H1N1): clinical features of pediatric hospitalizations in two successive waves. Int J Infect Dis 2011;15:e122-30.

16. Cox CM, Blanton L, Dhara R, Brammer L, Finelli L. 2009 Pandemic influenza A (H1N1) deaths among children: United States, 2009-2010. Clin Infect Dis 2011;52 Suppl 1:S69-74. 